TAMKANG JOURNAL OF MATHEMATICS

Volume 33, Number 1, Spring 2002

\title{
ON NONLINEAR FINITE DIFFERENCE INEQUALITIES IN TWO INDEPENDENT VARIABLES
}

\author{
B. G. PACHPATTE
}

\begin{abstract}
The aim of the present paper is to establish some new finite difference inequalities involving functions of two independent variables which provide explicit bounds on unknown functions. The inequalities given here can be used as tools in the qualitative theory of certain partial finite difference equations.
\end{abstract}

\section{Introduction}

In many cases when studying the behavior of solutions of certain classes of finite difference equations we need some specific type of finite difference inequalities. In [1-6], the authors have investigated some new finite difference inequalities which are motivated and inspired from the study of certain classes of finite difference equations. In the present paper, we establish some new finite difference inequalities involving functions of two independent variables, which can be used as ready and powerful tools in the analysis of certain classes of partial finite difference and sum-difference equations.

\section{Statement of Results}

In what follows, $R$ denotes the set of real numbers and $R_{+}=[0, \infty), R_{1}=[1, \infty), N_{0}=$ $\{0,1,2, \cdots\}$ are the given subsets of $R$. We use the usual conventions that empty sums and products are taken to be 0 and 1 respectively and assume that all the sums and products involved throughtout the discussion exist on the respective domains of their definitions.

Our main results are given in the following theorems.

Theorem 1. Let $u(m, n), f(m, n)$ be real-valued nonnegative functions defined for $m, n \in N_{0}$ and $c \geq 0$ be a constant.

Received March 22, 2001.

2000 Mathematics Subject Classification. 26D15, 26D20.

Key words and phrases. Nonlinear, finite difference inequalities, two independent variables, partial finite difference equations, empty sums and products, nondecreasing, nonincreasing, explicit bound, sum-difference equation. 
$\left(a_{1}\right)$ If

$$
u^{2}(m, n) \leq c+\sum_{s=0}^{m-1} \sum_{t=n+1}^{\infty} f(s, t) u(s, t)
$$

for $m, n \in N_{0}$, then

$$
u(m, n) \leq \sqrt{c}+\frac{1}{2} \sum_{s=0}^{m-1} \sum_{t=n+1}^{\infty} f(s, t)
$$

for $m, n \in N_{0}$.

$\left(a_{2}\right)$ Let $W$ be a real-valued continuous, nondecreasing function defined on $R_{+}$. Take, $W>0$ on $(0, \infty)$, and $W(0)=0$. If

$$
u^{2}(m, n) \leq c+\sum_{s=0}^{m-1} \sum_{t=n+1}^{\infty} f(s, t) u(s, t) W(u(s, t)),
$$

for $m, n \in N_{0}$, then for $0 \leq m \leq m_{1}, 0 \leq n \leq n_{1}, m, m_{1}, n, n_{1} \in N_{0}$,

$$
u(m, n) \leq G^{-1}\left[G(\sqrt{c})+\frac{1}{2} \sum_{s=0}^{m-1} \sum_{t=n+1}^{\infty} f(s, t)\right],
$$

where

$$
G(r)=\int_{r_{0}}^{r} \frac{d s}{W(s)}, r>0, r_{0}>0
$$

$G^{-1}$ is the inverse function of $G$ and

$$
G(\sqrt{c})+\frac{1}{2} \sum_{s=0}^{m-1} \sum_{t=n+1}^{\infty} f(s, t) \in \operatorname{Dom}\left(G^{-1}\right),
$$

for $0 \leq m \leq m_{1}, 0 \leq n \leq n_{1}, m, m_{1}, n, n_{1} \in N_{0}$.

$\left(a_{3}\right)$ Let $A: N_{0}^{2} \times R_{+} \rightarrow R_{+}$be a function which satisfies the condition

$$
0 \leq A(m, n, u)-A(m, n, v) \leq B(m, n, v)(u-v),
$$

for $u \geq v \geq 0$, where $B(m, n, v)$ is a real-valued nonnegative function defined for $m, n \in N_{0}, v \in R_{+}$. If

$$
u^{2}(m, n) \leq c+\sum_{s=0}^{m-1} \sum_{t=n+1}^{\infty} f(s, t) u(s, t) A(s, t, u(s, t)),
$$

for $m, n \in N_{0}$, then

$$
u(m, n) \leq \sqrt{c}+a(m, n) \prod_{s=0}^{m-1}\left[1+\sum_{t=n+1}^{\infty} \frac{1}{2} f(s, t) B(s, t, \sqrt{c})\right]
$$


for $m, n \in N_{0}$, where

$$
a(m, n)=\frac{1}{2} \sum_{s=0}^{m-1} \sum_{t=n+1}^{\infty} f(s, t) A(s, t, \sqrt{c}),
$$

for $m, n \in N_{0}$.

Theorem 2. Let $u(m, n)$ be a function defined on $N_{0}^{2}$ into $R_{1}, f(m, n)$ be a function as defined in Theorem 1 and $c \geq 1$ be a constant.

$\left(b_{1}\right)$ If

$$
u(m, n) \leq c+\sum_{s=0}^{m-1} \sum_{t=n+1}^{\infty} f(s, t) u(s, t) \log u(s, t),
$$

for $m, n \in N_{0}$, then

$$
u(m, n) \leq c^{P(m, n)},
$$

for $m, n \in N_{0}$, where

$$
P(m, n)=\prod_{s=0}^{m-1}\left[1+\sum_{t=n+1}^{\infty} f(s, t)\right]
$$

for $m, n \in N_{0}$.

$\left(b_{2}\right)$ Let $W$ be as defined in Theorem 1 (part $\left.\left(a_{2}\right)\right)$. If

$$
u(m, n) \leq c+\sum_{s=0}^{m-1} \sum_{t=n+1}^{\infty} f(s, t) u(s, t) W(\log u(s, t)),
$$

for $m, n \in N_{o}$, then for $0 \leq m \leq m_{2}, 0 \leq n \leq n_{2}, m, m_{2}, n, n_{2} \in N_{0}$,

$$
u(m, n) \leq \exp \left[G^{-1}\left[G(\log c)+\sum_{s=0}^{m-1} \sum_{t=n+1}^{\infty} f(s, t)\right]\right],
$$

where $G, G^{-1}$ are as defined in Theorem $1\left(\left(\right.\right.$ parat $\left.\left(a_{2}\right)\right)$ and

$$
G(\log c)+\sum_{s=0}^{m-1} \sum_{t=n+1}^{\infty} f(s, t) \in \operatorname{Dom}\left(G^{-1}\right),
$$

for $0 \leq m \leq m_{2}, 0 \leq n \leq n_{2}, m, m_{2}, n, n_{2} \in N_{0}$.

$\left(b_{3}\right)$ Let $A, B$ be the functions as defined in Theorem 1 (part $\left.\left(a_{3}\right)\right)$ which satisfies the condition (2.6). If

$$
u(m, n) \leq c+\sum_{s=0}^{m-1} \sum_{t=n+1}^{\infty} f(s, t) u(s, t) A(s, t, \log u(s, t)),
$$


for $m, n \in N_{0}$, then

$$
u(m, n) \leq c \exp \left[b(m, n) \prod_{s=0}^{m-1}\left[1+\sum_{t=n+1}^{\infty} f(s, t) B(s, t, \log c)\right]\right]
$$

for $m, n \in N_{0}$, where

$$
b(m, n)=\sum_{s=0}^{m-1} \sum_{t=n+1}^{\infty} f(s, t) A(s, t, \log c),
$$

for $m, n \in N_{0}$.

\section{Proof of Theorem 1}

$\left(a_{1}\right)$ We first assume that $c>0$ and define a function $z(m, n)$ by the right side of (2.1), then $u(m, n) \leq \sqrt{z(m, n)}$ and

$$
\begin{aligned}
& {[z(m+1, n)-z(m, n)]-[z(m+1, n+1)-z(m, n+1)] } \\
= & f(m, n+1) u(m, n+1) \\
\leq & f(m, n+1) \sqrt{z(m, n+1)} .
\end{aligned}
$$

By using the facts that $\sqrt{z(m, n)}>0, \sqrt{z(m, n+1)} \leq \sqrt{z(m, n)}, \sqrt{z(m, n+1)} \leq$ $\sqrt{z(m+1, n+1)}, \sqrt{z(m+1, n+1)} \leq \sqrt{z(m+1, n)}$, we observe that

$$
\begin{aligned}
& {[\sqrt{z(m+1, n)}-\sqrt{z(m, n)}]-[\sqrt{z(m+1, n+1)}-\sqrt{z(m, n+1)}] } \\
= & \frac{[z(m+1, n)-z(m, n)]}{\sqrt{z(m+1, n)}+\sqrt{z(m, n)}}-\frac{[z(m+1, n+1)-z(m, n+1)]}{\sqrt{z(m+1, n+1)}+\sqrt{z(m, n+1)}} \\
\leq & \frac{[z(m+1, n)-z(m, n)]}{\sqrt{z(m+1, n+1)}+\sqrt{z(m, n+1)}}-\frac{[z(m+1, n+1)-z(m, n+1)]}{\sqrt{z(m+1, n+1)}+\sqrt{z(m, n+1)}} \\
= & \frac{[z(m+1, n)-z(m, n)]-[z(m+1, n+1)-z(m, n+1)]}{\sqrt{z(m+1, n+1)}+\sqrt{z(m, n+1)}} \\
\leq & \frac{[z(m+1, n)-z(m, n)]-[z(m+1, n+1)-z(m, n+1)]}{\sqrt{z(m, n+1)}+\sqrt{z(m, n+1)}} \\
\leq & \frac{1}{2} f(m, n+1) .
\end{aligned}
$$

Here we have used (3.1) to get (3.2). Now keeping $m$ fixed in (3.2), set $n=t$ and sum over $t=n, n+1, \cdots, q-1\left(q \geq n+1\right.$ is arbitrary in $\left.N_{0}\right)$ to obtain

$$
[\sqrt{z(m+1, n)}-\sqrt{z(m, n)}]-[\sqrt{z(m+1, q)}-\sqrt{z(m, q)}] \leq \frac{1}{2} \sum_{t=n+1}^{q} f(m, t) .
$$


Noting that $\lim _{q \rightarrow \infty} \sqrt{z(m+1, q)}=\lim _{q \rightarrow \infty} \sqrt{z(m, q)}=\sqrt{c}$, and by letting $q \rightarrow \infty$ in (3.3) we get

$$
\sqrt{z(m+1, n)}-\sqrt{z(m, n)} \leq \frac{1}{2} \sum_{t=n+1}^{\infty} f(m, t) .
$$

Keeping $n$ fixed in (3.4), set $m=s$ and sum over $s=0,1,2, \cdots, m-1$, and use the fact that $z(0, n)=c$, to obtain

$$
\sqrt{z(m, n)} \leq \sqrt{c}+\frac{1}{2} \sum_{s=0}^{m-1} \sum_{t=n+1}^{\infty} f(s, t)
$$

The desired inequality in (2.2) follows by using the fact that $u(m, n) \leq \sqrt{z(m, n)}$. If $c \geq 0$, we carry out the above procedure with $c+\epsilon$ instead of $c$, where $\epsilon>0$ is an arbitrary small constant, and subsequently pass to the limit as $\epsilon \rightarrow 0$ to obtain (2.2).

$\left(a_{2}\right)$ Let $c>0$ and define a function $z(m, n)$ by the right side of $(2.3)$. Then, by following the same arguments as in the proof of $\left(a_{1}\right)$ upto (3.2) with suitable changes we get

$$
\begin{aligned}
& {[\sqrt{z(m+1, n)}-\sqrt{z(m, n)}]-[\sqrt{z(m+1, n+1)}-\sqrt{z(m, n+1)}] } \\
\leq & \frac{1}{2} f(m, n+1) W(\sqrt{z(m, n+1)}) .
\end{aligned}
$$

From (3.6) and using the fact that $W(\sqrt{z(m, n+1)}) \leq W(\sqrt{z(m, n)})$ we observe that

$$
\begin{aligned}
& \frac{[\sqrt{z(m+1, n)}-\sqrt{z(m, n)}]}{W(\sqrt{z(m, n)})}-\frac{[\sqrt{z(m+1, n+1)}-\sqrt{z(m, n+1)}}{W(\sqrt{z(m, n+1)})} \\
\leq & \frac{1}{2} f(m, n+1) .
\end{aligned}
$$

Keeping $m$ fixed in (3.7), set $n=t$ and sum over $t=n, n+1, \cdots, q-1(q \geq n+1$ is arbitrary in $N_{0}$ ) to obtain the estimate

$$
\frac{[\sqrt{z(m+1, n)}-\sqrt{z(m, n)}]}{W(\sqrt{z(m, n)})}-\frac{[\sqrt{z(m+1, q)}-\sqrt{z(m, q)}}{W(\sqrt{z(m, q)})} \leq \frac{1}{2} \sum_{t=n+1}^{q} f(m, t) .
$$

Noting that $\lim _{q \rightarrow \infty} \sqrt{z(m+1, q)}=\lim _{q \rightarrow \infty} \sqrt{z(m, q)}=\sqrt{c}$ and by letting $q \rightarrow \infty$ in (3.8) we get

$$
\frac{[\sqrt{z(m+1, n)}-\sqrt{z(m, n)}]}{W(\sqrt{z(m, n)})} \leq \frac{1}{2} \sum_{t=n+1}^{\infty} f(m, t) .
$$

From (2.5) and (3.9) we have

$$
G(\sqrt{z(m+1, n)})-G(\sqrt{z(m, n)})=\int_{\sqrt{z(m, n)}}^{\sqrt{z(m+1, n)}} \frac{d s}{W(s)}
$$




$$
\begin{aligned}
& \leq \frac{[\sqrt{z(m+1, n)}-\sqrt{z(m, n)}]}{W(\sqrt{z(m, n)})} \\
& \leq \frac{1}{2} \sum_{t=n+1}^{\infty} f(m, t) .
\end{aligned}
$$

Now Keeping $n$ fixed in (3.10), set $m=s$ and sum both sides over $s=0,1,2, \cdots, m-1$ and use the fact that $z(0, n)=c$ to obtain

$$
G(\sqrt{z(m, n)}) \leq G(\sqrt{c})+\frac{1}{2} \sum_{s=0}^{m-1} \sum_{t=n+1}^{\infty} f(s, t) .
$$

The required inequality in (2.4) follows from (3.11) and $u(m, n) \leq \sqrt{z(m, n)}$. The case $c \geq 0$ can be completed as mentioned in the proof of part $\left(a_{1}\right)$. The subdomain $0 \leq m \leq m_{1}, 0 \leq n \leq n_{1}$ is obvious.

$\left(a_{3}\right)$ Let $c>0$ and define a function $z(m, n)$ by the right side of $(2.7)$. Then $u(m, n) \leq$ $\sqrt{z(m, n)}$ and by following the proof of $\left(a_{1}\right)$ upto (3.2) with suitable changes we get

$$
\begin{aligned}
& {[\sqrt{z(m+1, n)}-\sqrt{z(m, n)}]-[\sqrt{z(m+1, n+1)}-\sqrt{z(m, n+1)}] } \\
\leq & \frac{1}{2} f(m, n+1) A(m, n+1, \sqrt{z(m, n+1)}) .
\end{aligned}
$$

Further by following the arguments as in the proof of $\left(a_{1}\right)$ below (3.2) upto (3.5) with suitable changes we get

$$
\sqrt{z(m, n)} \leq \sqrt{c}+\frac{1}{2} \sum_{s=0}^{m-1} \sum_{t=n+1}^{\infty} f(s, t) A(s, t, \sqrt{z(s, t)}) .
$$

Define a function $v(m, n)$ by

$$
v(m, n)=\frac{1}{2} \sum_{s=0}^{m-1} \sum_{t=n+1}^{\infty} f(s, t) A(s, t, \sqrt{z(s, t)}) .
$$

From (3.14) and (2.6) we observe that

$$
\begin{aligned}
v(m, n) \leq & \frac{1}{2} \sum_{s=0}^{m-1} \sum_{t=n+1}^{\infty} f(s, t)[A(s, t, \sqrt{c}+v(s, t))-A(s, t, \sqrt{c})] \\
& +\frac{1}{2} \sum_{s=0}^{m-1} \sum_{t=n+1}^{\infty} f(s, t) A(s, t, \sqrt{c}) \\
\leq & a(m, n)+\frac{1}{2} \sum_{s=0}^{m-1} \sum_{t=n+1}^{\infty} f(s, t) B(s, t, \sqrt{c}) v(s, t),
\end{aligned}
$$


where $a(m, n)$ is defined by $(2.9)$. Clearly $a(m, n)$ is nondecreasing in $m$ and nonincreasing in $n$. An application of Theorem 1 part $\left(a_{2}\right)$ given in [5] to (3.15) yields

$$
v(m, n) \leq a(m, n) \prod_{s=0}^{m-1}\left[1+\sum_{t=n+1}^{\infty} \frac{1}{2} f(s, t) B(s, t, \sqrt{c})\right] .
$$

The required inequality in (2.8) follows by using the fact that $u(m, n) \leq \sqrt{z(m, n)}$ and (3.13). The proof of the case when $c \geq 0$ can be completed as mentioned in the proof of $\left(a_{1}\right)$.

\section{Proof of Theorem 2}

$\left(b_{1}\right)$ Define a function $z(m, n)$ by the right side of $(2.10)$, then $u(m, n) \leq z(m, n)$ and

$$
\begin{aligned}
& {[z(m+1, n)-z(m, n)]-[z(m+1, n+1)-z(m, n+1)] } \\
= & f(m, n+1) u(m, n+1) \log u(m, n+1) \\
\leq & f(m, n+1) z(m, n+1) \log z(m, n+1) .
\end{aligned}
$$

From the definition of $z(m, n)$ we see that $z(m, n+1) \leq z(m, n)$ for $m, n \in N_{0}$. Using this fact, from (4.1) we observe that

$$
\begin{aligned}
& \frac{[z(m+1, n)-z(m, n)]}{z(m, n)}-\frac{[z(m+1, n+1)-z(m, n+1)]}{z(m, n+1)} \\
\leq & f(m, n+1) \log z(m, n+1) .
\end{aligned}
$$

Keeping $m$ fixed in (4.2), set $n=t$ and sum over $t=n, n+1, \cdots, q-1(q \geq n+1$ is arbitrary in $N_{0}$ ) to obtain the estimate

$$
\begin{aligned}
& \frac{[z(m+1, n)-z(m, n)]}{z(m, n)}-\frac{[z(m+1, q)-z(m, q)]}{z(m, q)} \\
\leq & \sum_{t=n+1}^{q} f(m, t) \log z(m, t) .
\end{aligned}
$$

Noting that $\lim _{q \rightarrow \infty} z(m+1, q)=\lim _{q \rightarrow \infty} z(m, q)=c$ and by letting $q \rightarrow \infty$ in (4.3) we get

$$
z(m+1, n) \leq\left[1+\sum_{t=n+1}^{\infty} f(m, t) \log z(m, t)\right] z(m, n) .
$$

Now keeping $n$ fixed in (4.4) and setting $m=s$ and then substituting $s=0,1,2, \cdots, m-1$ successively and using the fact that $z(0, n)=c$, we obtain the estimate

$$
\begin{aligned}
z(m, n) & \leq c \prod_{s=0}^{m-1}\left[1+\sum_{t=n+1}^{\infty} f(s, t) \log z(s, t)\right] \\
& \leq c \exp \left(\sum_{s=0}^{m-1} \sum_{t=n+1}^{\infty} f(s, t) \log z(s, t)\right) .
\end{aligned}
$$


From (4.5) we observe that

$$
\log z(m, n) \leq \log c+\sum_{s=0}^{m-1} \sum_{t=n+1}^{\infty} f(s, t) \log z(s, t) .
$$

From (4.6) and following the same arguments as above with suitable changes we obtain the estimate

$$
\begin{aligned}
\log z(m, n) & \leq \log c\left[\prod_{s=0}^{m-1}\left[1+\sum_{t=n+1}^{\infty} f(s, t)\right]\right] \\
& =\log c^{P(m, n)}
\end{aligned}
$$

where $P(m, n)$ is defined by (2.12). From (4.7) we observe that

$$
z(m, n) \leq c^{P(m, n)} .
$$

Using (4.8) in $u(m, n) \leq z(m, n)$ we get the required inequality in (2.11).

The details of the proofs of $\left(b_{2}\right)\left(b_{3}\right)$ can be completed by closely looking at the proofs of $\left(a_{2}\right),\left(a_{3}\right)$ and $\left(b_{1}\right)$, see, also $[3,4,5]$. We omit the details.

\section{An Application}

In this section we present an immediate application of Theorem 2 part $\left(b_{1}\right)$ to obtain an explicit bound on solution of the following sum-difference equation

$$
u(m, n)=e(m, n)+\sum_{s=0}^{m-1} \sum_{t=n+1}^{\infty} h(s, t) u(s, t) \log |u(s, t)|,
$$

where $u(m, n), e(m, n), h(m, n)$ are real-valued functions defined for $m, n \in N_{0}$ and $|e(m, n)| \leq c$, where $c \geq 0$ is a constant. Let $u(m, n)$ be a solution of (5.1) defined for $m, n \in N_{0}$. From (5.1) we observe that

$$
1+|u(m, n)| \leq 1+c+\sum_{s=0}^{m-1} \sum_{t=n+1}^{\infty}|h(s, t)|(1+|u(s, t)|) \log (1+|u(s, t)|) .
$$

Now a suitable application of Theorem 2, part $\left(b_{1}\right)$ to $(5.2)$ yields

$$
|u(m, n)| \leq\left[(1+c)^{Q(m, n)}-1\right]
$$

where

$$
Q(m, n)=\prod_{s=0}^{m-1}\left[1+\sum_{t=n+1}^{\infty}|h(s, t)|\right]
$$


for $m, n \in N_{0}$. The inequality (5.3) obtains the bound on the solution $u(m, n)$ of (5.1) in terms of the known functions.

We note that the inequality given in Theorem 1, part $\left(a_{1}\right)$ can be used to obtain bound on solution of the following sum-difference equation

$$
u^{2}(m, n)=e(m, n)+\sum_{s=0}^{m-1} \sum_{t=n+1}^{\infty} F(s, t, u(s, t)),
$$

under some suitable conditions on the functions involved in (5.5). Various other applications of the inequalities given here will be reported elsewhere.

\section{References}

[1] F. S. De Blasi and J. Schinas, On the stability of difference equations in Banach spaces, An. Sti. Univ. "Al. I. Cuza" Iasi. Sect. I, 20 (1974), 65-80.

[2] A. Mate and P. Nevai, Sublinear perturbations of the differential equation $y^{(n)}=0$ and of the analogous difference equation, J. Differential Equations, 53(1984), 234-257.

[3] B. G. Pachpatte, On certain new finite difference inequalities, Indian J. Pure Appl. Math., 24(1993), 373-384.

[4] B. G. Pachpatte, Inequalities for Finite Difference Equations, Marcel Dekker, Inc. New York, 2001.

[5] B. G. Pachpatte, On some finite difference inequalities in two independent variables, J. Math. Anal. Appl., 254(2001), 587-598.

[6] G. Papaschinopoules, On the summable manifold for discrete systems, Math. Japonica, 33(1988), 457-468.

57 Shri Niketan Colony, Aurangabad 431 001, (Maharashtra) India. 